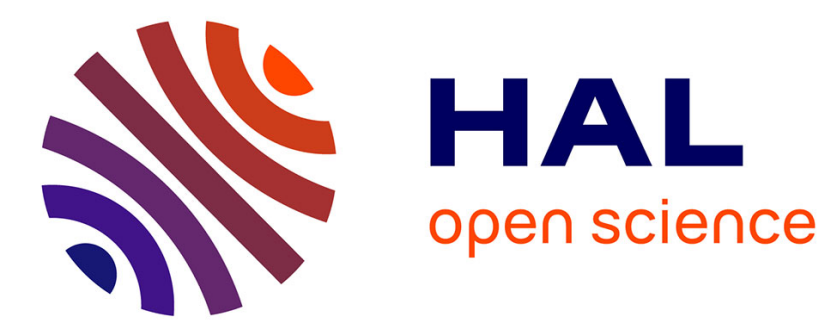

\title{
Intégrer les sites web dans les stratégies: concept et modèle
}

Marie-Christine Longe Monnoyer, Catherine Lapassouse Madrid

\section{To cite this version:}

Marie-Christine Longe Monnoyer, Catherine Lapassouse Madrid. Intégrer les sites web dans les stratégies: concept et modèle. Revue Française de Gestion, 2007, 10.3166/rfg.173.145-155 hal-02616697

\section{HAL Id: hal-02616697 \\ https://hal.science/hal-02616697}

Submitted on 24 May 2020

HAL is a multi-disciplinary open access archive for the deposit and dissemination of scientific research documents, whether they are published or not. The documents may come from teaching and research institutions in France or abroad, or from public or private research centers.
L'archive ouverte pluridisciplinaire HAL, est destinée au dépôt et à la diffusion de documents scientifiques de niveau recherche, publiés ou non, émanant des établissements d'enseignement et de recherche français ou étrangers, des laboratoires publics ou privés. 


\section{Intégrer les sites web dans les stratégies: concept et modèle RFG $\mathbf{N}^{\circ} 173$ avril 2007}

Marie-Christine MONNOYER, IAE Université Toulouse I

Place A. France

31042 Toulouse, France

marie-christine.monnoyer @ univ-tlse1.fr
Catherine LAPASSOUSE MADRID

IUT Bordeaux Montesquieu

Département Techniques de Commercialisation

Avenue Abadie, 33000 Bordeaux catherine.madrid@wanadoo.fr

Résumé

Some firm's observations lead The authors to formulate a new conceptual frame to analyse how firms can shape their strategy and their marketing analysis according to their web site. From there, they propose a model which helps managers to understand how deep the integration goes

S'appuyant sur les principales avancées conceptuelles portant sur l'intégration des TIC dans le domaine de la réflexion stratégique et de la démarche marketing, en sur de nombreuses analyses terrain, les auteures ont défini un cadre conceptuel synthétique relatif à l'intégration d'un site web dans l'organisation et la stratégie commerciale d'une entreprise. Cette démarche leur permet de proposer un modèle d'analyse de cette intégration qui permet aux managers de prendre la mesure des qualités ou des faiblesses de cette intégration dans leur entreprise. 
L'enquête annuelle BNP Paribas de mars 2006 fait état d'une croissance de 50\% des investissements des entreprises dans le domaine des technologies de l'information et de la communication (T.I.C) en France au cours des 12 mois précédents. Ce dynamisme suppose que l'ensemble des entreprises et non plus les seuls «dot com»comprenne et maîtrise les leviers de la compétitivité commerciale numérique. Or, cette problématique dépasse le seul champ de la stratégie marketing. La mise en réseau des informations autorise, en effet, des révolutions organisationnelles et stratégiques de première importance pour toutes les entreprises (Scott Morton 1990), Nous ferons donc le point sur les avancées, de la réflexion stratégique et de la démarche marketing, portant sur l'intégration des TIC. Plus concrètement, nous mettrons en valeur les effets de ces apports en nous appuyant sur le cas de la PME PH distributeur d'articles sportifs,spécialisé dans le sport de glace. Le site marchand, créé six mois plus tôt, avait permis d'augmenter les ventes de $25 \%$. Ce succès surchargeait de travail (logistique et mise à jour du site) le dirigeant fondateur du magasin, mais ne lui permettait pas d'engager du personnel, Quelles recommandations stratégiques et/ou organisationnelles pouvions nous formuler? Nous avons finalisé un outil d'analyse des sites, applicables aux PME, qui intègre et rend lisible le potentiel de l'intégration des TIC et les écarts existant par rapport à sa propre situation.

\section{Encadré méthodologique}

Notre recherche terrain s'appuie sur deux modes de recueil d'informations : des interviews de dirigeants cadres ou employés d'entreprises (50 entretiens menés par deux chercheurs à partir d'un questionnaire ouvert, enregistrés et retranscrits) et une analyse des sites électroniques marchands et non marchands (environ 150 sites suivis). M. Hlady-Rispal (2001) parle d'un " mouvement de balancier" permanent entre l'observation et l'abstraction pour décrire la démarche du chercheur entre les domaines théorique et opératoire. C'est celuici qui nous conduit à revisiter les sites avec de nouvelles grilles en fonction de notre progression théorique. La mise au point du modèle a été affinée après plusieurs tests effectués en ligne et hors ligne avec les responsables intéressés.

\section{Le concept « d'alignement stratégique » versus « l’approche outil »}

Les travaux en sciences de gestion menés dans le prolongement du développement commercial des TIC ont été initialement impulsés selon deux approches, celle des chercheurs en stratégie et en systèmes d'informations d'une part, celle des auteurs en marketing, d'autre part. On trouve en trame de fond de la 
confrontation des deux conceptions des T.I.C. un outil supplémentaire au service de la stratégie ou du marketing d'une part, un levier de renouvellement du management de l'entreprise d'autre part.

\section{- $\quad$ L’alignement stratégique une condition nécessaire ....}

Si la dimension stratégique de l'utilisation des TIC est relevée très tôt par les praticiens et les chercheurs (Mc Farlan, 1983 ; Porter et Millar, 1986), Henderson et Venkatraman (1999) soulignent le caractère non automatique de l'usage des TIC sur l'évolution des orientations stratégiques. Ce sont les apports de la théorie de la contingence qui vont conduire ces derniers auteurs et leurs successeurs, tels Raymond et Bergeron (2001) à annoncer que les stratégies d'investissement en TIC doivent être co-alignées avec les comportements stratégiques généraux de l'entreprise pour en affecter positivement la performance. Ces technologies peuvent être porteuses d'avantages concurrentiels, de flexibilité stratégique, si et seulement si un processus de migration vers « l’entreprise numérique » a été entrepris (Krell et Gale 2001).

Les TIC n'apparaissent plus seulement comme un outil au service de la stratégie, mais comme les déclencheurs d'une dynamique qui peut initier des réflexions stratégiques. Derrière une potentialité se cache une contrainte qu'il faut expliquer pour la dépasser. Les apports de la théorie de la structuration et du modèle d'acceptation de la technologie y contribuent.

\section{- La réflexivité, catalyseur de l'alignement}

Parce que les TIC modifient les structures cognitives de l'organisation, les acteurs réagissent à leur introduction dans l'organisation. Cette réflexivité analysée par Giddens, dans la théorie de la structuration (1986) « est inhérente à l'action humaine..., elle est la façon spécifiquement humaine de contrôler le flux continu de la vie sociale » (1994).

La facilité d'utilisation de la technologie, qui inclut des dimensions subjectives propres à chaque utilisateur, l'utilité perçue, mais aussi le plaisir éprouvé du fait de l'usage apparaissent comme les vecteurs de l'acceptation d'une technologie et de la dynamique du comportement de l'usager (Davis, 1989, modèle 
d'acceptation de la technologie). Lorsque les actions réflexives des utilisateurs sont négatives, elles affaiblissent les potentialités factuelles des TIC. Lorsqu'elles sont positives, elles permettent d'exploiter au mieux ces potentialités et conduisent les acteurs à formuler des propositions stratégiques, quel que soit (ou presque) le niveau et le champ des responsabilités des acteurs. Ces concepts nous permettent de comprendre pourquoi seule la congruence (terme choisi par Raymond), entre gestion des TIC et orientation stratégique, est susceptible d'exploiter les effets positifs des actions réflexives nées des usages des T.I.C. et de pallier, dans une certaine mesure, les effets négatifs de ces actions qui peuvent aller jusqu'à l'annihilation des choix stratégiques.

Dans le cas de PH il n'y a pas de congruence. La décision de création d'un site a été prise parce qu'elle était d'actualité et que la culture personnelle du dirigeant le sensibilisait à l'usage des TIC. La stratégie de l'entreprise n'a pas été « revisitée » après cette installation.

\section{- $\quad$ Site web et co-alignement}

La focalisation de notre attention sur le site web conduit à relever que son implantation peut être le fruit d'un effet de mode. Mais, son développement dans le temps, sa mise à jour traduisent un comportement pro-actif des dirigeants, voire une stratégie de prospection ou d'analyse du marché (au sens donné par Miles et Snow 1978). Toutefois, parce qu'il modifie les structures cognitives de l'entreprise, le site a un effet sur l'organisation du travail. Il peut modifier la perception des membres de l'entreprise sur leur organisation. Il constitue un nouveau lieu de servuction qui agit sur la qualité du service. Il peut même apparaître comme une action de re-localisation (au sens de Giddens) de la relation au client ou au fournisseur, si la communication numérisée réduit les erreurs et génère de la confiance.

Le site web apparaît donc porteur de ressources organisationnelles et stratégiques qui ne se révèlent que dans le cadre d'une congruence entre gestion des TIC et choix stratégiques. Les potentialités d'innovation qu'apporte le site, exacerbent les besoins d'intégration et de coordination entre les processus productifs, relevés par Lawrence et Lorsch (1967) dans l'analyse des entreprises performantes. Nous retiendrons qu'un 
modèle d'analyse des sites doit faire apparaître les déficiences ou les réussites en matière de coordination et d'intégration.

\section{- Une conception marketing initiale restrictive}

Dans la littérature marketing, l'analyse des incidences stratégiques donne lieu à des réflexions différentes selon que l'on considère le site comme un outil au service de la politique de communication, ou de la distribution (Kotler, 2003, Lendrevie, 2003).

L’interactivité procurée par le site et la personnalisation des contacts qui peuvent en résulter, ont fait prendre conscience de ses possibilités d'application en communication soit comme une enrichissement du marketing direct soit comme un média supplémentaire (Torger, 2006). Elle introduit une dimension que Notebaert (1986) qualifie « de lien social », vecteur susceptible de renforcer la différenciation de l'entreprise grâce à la personnalisation de la relation.

L'ouverture d'un site marchand correspond quant à elle à l'adjonction d'un canal supplémentaire dans un circuit de distribution (points de vente physique), qualifié de «système de distribution dual» (Vanheems, 2004). Cet élargissement fait naître des problèmes de transferts de clientèles (Vanheems 2004) ou des conflits intra-canaux (Lendrevie et al. 2003)

L'analyse des principaux manuels de référence montre que les incidences de l'usage d'Internet sont traitées de manière morcelée, à chaque stade opérationnel de la stratégie commerciale (Kotler 2003), l'approche « outil au service du mix » est patente.

Le cas PH illustre cette conception « outil » : le responsable n'a pas considéré les incidences de son site de manière globale sur sa stratégie commerciale. Il est dépassé par son succès.

\section{- Vers un renouvellement de l'approche marché}

Dubois et Vernette proposaient la définition suivante du « e marketing » en 2001 : « mobilisation par le 
marketing de toutes les dimensions du potentiel technologique offert par les nouvelles technologies pour une approche renouvelée des marchés de l'entreprise ». Cette conception du rôle des T.I.C. en tant que levier du développement commercial rejoint - au stade de l'analyse marketing -les réflexions des chercheurs en stratégie sur le besoin d'alignement entre choix des TIC et décisions stratégiques.

L'approche outil, apparaît en revanche, en contradiction avec cette perspective. Dans cette approche, l'entreprise n'invente rien, elle propose seulement, autrement. Sa compétitivité s'appuie dès lors, sur son degré de maîtrise de la numérisation des fonctions de communication et/ou de commercialisation. Celle-ci ne s'affaiblira-t-il pas avec la diffusion de l'outil internet?

Sans véritable réflexion sur l'enrichissement de son offre numérique, l'entreprise PH est à la merci d'un concurrent qui ouvrirait un site marchand et pourrait ainsi attirer ses clients, quel que soit son lieu d'implantation physique.

Cette perspective semble d'autant plus probable que l'interactivité associée au site développe l'autonomie du consommateur. Le marketing inversé (Kotler, 2002), suggère de dépasser la relation personnalisée pour envisager un achat interactif, en d'autres termes une co-construction de l'offre avec le consommateur (Torger, 2006). L'approche outil nous semble, dès lors, très réductrice par rapport aux tentatives d'intégration du site au sein de l'offre.

\section{- Par une intégration de l'interactivité au sein de l'offre}

Plusieurs tentatives d'intégration de l'interactivité au niveau de l'offre ont été réalisées, proposant une approche plus globale que la seule référence aux applications d'Internet au sein des différents types d'actions marketing. (Lévy, 2000, Lendrevie et alii, 2003). Partant des caractéristiques de l'offre, les auteurs distinguent quatre types de potentiels liés à des avantages de coût ou de différenciation. Toutefois, les auteurs envisagent aussi une « offre numérique ne bénéficiant ni d'un avantage de coût ni de différenciation » qu'ils qualifient « d'impasse numérique ». Il nous semble que ce concept n'a pas lieu d'être car les TIC sont 
susceptibles de procurer un effet de levier, quelle que soit l'offre. Il s'agit d'inverser le raisonnement et de réfléchir à la manière dont la numérisation peut renouveler l'offre.

Le dirigeant de PH se plaçait sans doute implicitement dans une logique de type "impasse numérique ». Comment imaginer en effet que l'impact d'un site marchand puisse être aussi important pour commercialiser des produits qu'il faut essayer avant d'acheter. C'était oublier que la rareté de l'offre pèse sur la demande, et que son accessibilité la stimule...Le concept d'impasse numérique montre ainsi ses limites.

Le renouvellement de l'approche marché nous éloigne de la conception d'un site, outil de marketing. Mais les réflexions sur l'interactivité ne vont pas jusqu'à une reconstruction de l'offre à partir des opportunités ouvertes par les TIC. En évoquant l'appel de Venkatraman aux dirigeants sur la pertinence du choix de leurs partenaires, sur le renouvellement de leur «métier », il nous paraît nécessaire d'avoir « une action réflexive » sur les cadres conceptuels utilisés et de les enrichir pour faciliter la congruence TIC-stratégie.

Notre réponse à la société PH ne se limitera donc pas à des conseils relatifs à la présentation du site ni à son contenu, mais à un questionnement sur l'enrichissement de l'offre proposée et aux moyens du back office en permettant la réalisation et la mise à disposition. Pour rendre compréhensible ces questions, nous présenterons dans le point suivant notre cadre conceptuel d'intégration ainsi que son application au cas PH

\section{Un cadre conceptuel d'intégration : « La chaîne de valeur reconfigurée 》}

Nous venons de mettre en exergue :

-la notion d'effet de levier susceptible d'être procuré par une conception des TIC, cherchant à renouveler l'approche des marchés. Cet effet prend sa source dans la création de valeur que le site peut apporter au client (Kotler, 2002). L’intégration de l'interactivité se situe ainsi plus en amont au niveau de la décision marketing, à savoir au niveau du positionnement et non plus de la conception de variables relatives au mix. 
-l'importance de l'alignement et de la coordination entre les implantations TIC et les évolutions stratégiques. La décision de création ou de reconfiguration d'un site web ne peut être détachée des modalités organisationnelles liées aux choix stratégiques.

Après avoir explicité le lien entre l'introduction de la numérisation au niveau de l'offre et la création de valeur, nous proposerons de reconfigurer la chaîne de valeur portérienne et nous l'utiliserons pour construire un outil d'analyse des sites.

- La reconfiguration de la chaîne de valeur

Dans la chaîne portérienne, deux activités principales agissent sur la phase de commercialisation. Pour intégrer l'ouverture du système d'offre à la dimension numérique, nous en ajoutons une troisième : dans le cas d'un site marchand, la numérisation de l'échange marchand et dans le cas d'un site de communication, la numérisation de la relation

L'introduction de cette nouvelle activité principale présente deux avantages :

-Elle permet de mettre en évidence les relations qu'entretient la numérisation et ses constituants avec toutes les autres activités qu'elles soient principales ou de soutien. Il est ainsi plus aisé de pointer les faiblesses de l'intégration de la dimension numérique avec des activités dont la dimension stratégique n'échappe à personne.

Ainsi dans le cas de l'entreprise PH, le dirigeant, ancien champion d'Europe d'une spécialité très pratiquée dans son pays, est-il porteur d'une culture nationale qui l'a porté "naturellement » vers l'internet, et de valeurs qui imprègnent fortement le sport qu'il pratique. Ces éléments font partie de ce que Porter appelle " les infrastructures » de la firme. Le concepteur du site n'a pas jugé utile d'y faire référence... parce que le dirigeant est " modeste ", parce que l'objectif est de commercialiser des produits.... La liaison infrastructures, numérisation de l'échange a été sous-estimée. 
-Elle permet aussi en utilisant la méthode de décomposition des activités utilisées par Porter de faire apparaître les éléments qui la constituent et sont créateurs de valeur.

Figure 1 .La reconfiguration de la chaîne de valeur

\begin{tabular}{|l|l|l|l|l|}
\hline \multicolumn{2}{|c|}{ Infrastructure de la firme } & \multirow{2}{*}{ VALEUR } \\
\hline \multicolumn{2}{|c|}{ Gestion des ressources humaines } \\
\hline \multicolumn{2}{|c|}{ Développement technologique } \\
\hline \multicolumn{2}{|c|}{ Approvisionnement } \\
interne
\end{tabular}

- Les composantes des activités liées à la numérisation dans les chaînes de valeur reconfigurées

* Les composantes de l'activité « numérisation de la relation »

Nous nous plaçons ici dans l'hypothèse où l'achat en ligne n'est pas possible. Le site propose à l'internaute une relation numérisée pouvant conduire à l'achat dans ses formes classiques. Nous décrirons ensuite le passage à un échange marchand et les composantes complémentaires qu'il nous paraît nécessaire d'intégrer.

Nous subdivisons l'activité « numérisation de la relation » en trois composantes : l'interaction virtuelle, la dimension expérientielle et l'I supply chain ». Leur rôle a été mis en évidence par de nombreux auteurs, nos enquêtes et observations de sites nous conduisent à en repréciser les contours et à les scinder, en unités élémentaires.

○ L’interactivité est liée de manière intrinsèque à la nature du média électronique. Sa mise en perspective dans le contexte d'une politique d'offre peut renouveler la stratégie de la firme et son marché. Son rôle, à ce niveau, repose sur trois unités :

-L'accessibilité de l'information sur l'offre permet au consommateur de trouver une base de 
données structurée très explicative des caractères de l'offre en réponse à un mot écrit sur un moteur de recherche

- L'ergonomie du site : La valeur de l'offre ne suffit pas à capter le prospect. Celui-ci risque d'abandonner sa consultation, s'il doit « entièrement traverser le site » pour trouver les conseils qu'il recherche ou s'il ne trouve pas le moyen de transmettre ses interrogations.

- La relation individualisée: L'ergonomie du site constitue le premier support de la construction d'une relation individualisée avec le prospect. Elle suppose la mise en place de ressources humaines conscientes des attentes d'individualisation des internautes qui ne deviendront des clients qu'avec un passage dans les surfaces de vente. C'est sur cette ergonomie que s'appuie la pérennisation de la relation au client, juste après ou longtemps après l'achat.

○ $\quad \mathrm{La}$ « dimension expérientielle » : Son originalité par rapport aux variables du plan de communication traditionnel (Flores, Volle, 2005) en fait le facteur le plus largement susceptible de déclencher la visite du site. Elle comporte des apports aussi bien cognitifs qu'affectifs et dépend de trois éléments :

- La richesse du contenu en ligne : C'est son existence réelle ou supposée qui incite le consommateur à consulter le site. L'information diffusée ouvre sur des problématiques associées à l'univers des produits (protection de l'environnement, alimentation diététique, santé ......). Le consommateur retire de la consultation une expérience cognitive (acquisition de connaissances)-,ou affective ( produit ou service en lien avec ses valeurs personnelles). Elle peut aller jusqu'à la naissance d'un sentiment d'ouverture, d'accomplissement personnel.

- La composante servicielle : L'information diffusée peut être personnalisée si l'internaute le souhaite. Les éléments de conseils spécialisés mis à sa disposition sont d'un accès difficile en dehors du net.

- La proximité relationnelle : si la finalité de la relation au prospect reste la réalisation d'un 
achat dans un point de vente physique, l'absence de transaction monétaire immédiate renforce la liberté de la consultation. En l'absence de face à face, la proximité relationnelle peut se manifester à travers la personnalisation ou la rapidité de la réponse. Elle constitue un facteur susceptible d'accroître la différenciation du site (Notebaert 2005), pourtant peu utilisé.

○ L'I supply chain : Dans un site de communication, l'organisation logistique doit fournir des informations en termes d'accessibilité à l'offre, nous l'appelons dès lors « supply chain informative ». Sa performance contribue à nourrir la relation numérique via :

- L'information sur l'accès à l'offre : L'internaute doit pouvoir trouver sur le site la liste des points de vente proches de ses lieux de vie.

- L'information sur les stocks : Dans la logique d'une relation de communication, la problématique de la gestion des stocks doit être centrée sur les disponibilités précises des produits dans les points de vente considérés par l'internaute. La qualité de l'I supply chain suppose donc une liaison forte avec l'activité logistique tant interne qu'externe.

Le site PH étant marchand nous ne déclinerons pas son exemple à propos de la numérisation de la relation. 2.2.2 Les composantes de la numérisation de l'échange

La mise en œuvre de l'activité « numérisation de l'échange » suppose la maîtrise de trois composantes qui constituent, les points de différenciation entre la commercialisation traditionnelle et la commercialisation numérisée : l'interaction virtuelle, la création et gestion de la confiance et la mise en place d'une « supply chain » électronique. Pour chacune d'entre elles, nous ne précisons que les éléments qui viennent compléter la numérisation de la relation.

○ L'interaction virtuelle :

-La qualité de l'accessibilité de l'offre repose sur des choix pertinents de référencement du site. Elle suppose le développement de la notoriété de l'adresse électronique grâce aux opérations de communication hors ligne.

PH qui est une TPE, peut proposer de satisfaire les besoins d'équipement de sportifs très éloignés géographiquement, parce que le sport concerné, peu pratiqué ne dispose pas de rayons spécifiques dans les 
grands magasins spécialisés et n'en subit donc pas la concurrence. L'ouverture d'un site est l'outil d'une stratégie de prospection active, le contenu sera le reflet du positionnement marketing adopté

- La gestion personnalisée de la relation : Le passage à une relation marchande virtuelle rend complexe la vérification de la compréhension des demandes et des informations transmises (par l'internaute, le site ou le webmaster) et exacerbe le besoin de confiance. Personnaliser la relation résout pour partie cette question. C'est aussi le moyen de différencier l'offre comme l'ont souligné la plupart des auteurs du « one to one ».

La volonté de s'attacher une clientèle éloignée a incité PH à mettre en ligne des outils de mesure de la taille et de l'adaptation fine des équipements aux spécificités corporelles de l'acheteur. Ils permettent d'accompagner le client dans son choix et de compenser l'absence physique du vendeur.

○ Les supports de confiance : Cette composante dont nous avons vu apparaitre l'intensité du fait du passage à une relation virtualisée et à la manipulation de systèmes abstraits peut s'appuyer sur quatre unités : les modalités de règlement, l'image de marque du site, l'interface de vente et la composante servicielle de l'offre.

- Les modalités de règlement : Au-delà du pré-requis de la sécurisation des paiements, le développement d'un discours clair sur les éléments qui composent le prix (frais de port...), la proposition de règlements hors ligne (chèque, à réception...) permettent de répondre aux besoins de confiance.

- L’image de marque du site : C'est un élément majeur pour communiquer sur l'offre et les valeurs auxquelles se réfère l'entreprise (Variot, 2001). En fournissant des repères aux internautes, l'image de marque du site va simplifier la réflexion, engendrer confiance et fidélisation.

- Le choix d'une interface de vente mixte : Il apporte à l'achat virtuel une dimension matérielle qui rassure.

- La composante servicielle : La numérisation des prestations telles la prise de commande, la facturation, l'aide à la sélection (via des conseils d'utilisation adaptés aux besoins de l'internaute), apparaît comme la source de services complémentaires. Ces prestations favorisant une «proximité 
virtuelle » sont créatrices de confiance. Elles contribuent à « mettre en scène » la valeur associée à l'offre.

Dès la première page du site de $\mathrm{PH}$, des modalités de règlement diversifiées apparaissent, la visibilité de la boutique sur le site affiche la possibilité d'une rencontre physique, de conseils personnalisés et rassure l'internaute qui réalise un achat important.

Les prestations de service supposent un travail de back office très important, qui ne peut être réalisé sans connaissances en informatique, contrainte sous-estimée par PH, qui renvoie sur l'activité « ressources humaines ».

○ L'e supply chain Dans un contexte de vente directe numérisée, l'organisation logistique doit accomplir deux des fonctions traditionnellement dévolues à un canal de distribution : la mise à disposition de l'offre et la gestion des stocks.

-La mise à disposition de l'offre : La commodité, la fiabilité et la rapidité de la livraison constituent un enjeu capital pour la position concurrentielle du e commerce face aux autres modes de distribution.

-La gestion des stocks : Dans la logique d'une relation client très personnalisée, la gestion des stocks doit, pour les références les plus significatives, rendre exceptionnelle la rupture.

La démarche de type portérien que nous avons menée, a permis d'enrichir la réflexion sur la structure des activités fonctionnelles et la nature des liens existant entre la numérisation de l'échange ou de la relation, leurs composantes et chacune des activités principales ou fonctionnelles. Elle fait apparaître l'importance de l'acceptation de ces nouvelles activités par les acteurs concernés dans l'entreprise, comme l'annonçaient les analyses issues des travaux de Giddens.

* Vers une application opérationnelle.

Ce cadre conceptuel présente l'intérêt de permettre la construction d'un outil de mesure de l'intégration d'un site dans la stratégie et l'organisation (O.N.I.S.M.O) (Madrid et Monnoyer, 2005). 
Figure 2. Modèle O.N.I.S.M.O. appliqué à l'entreprise PH

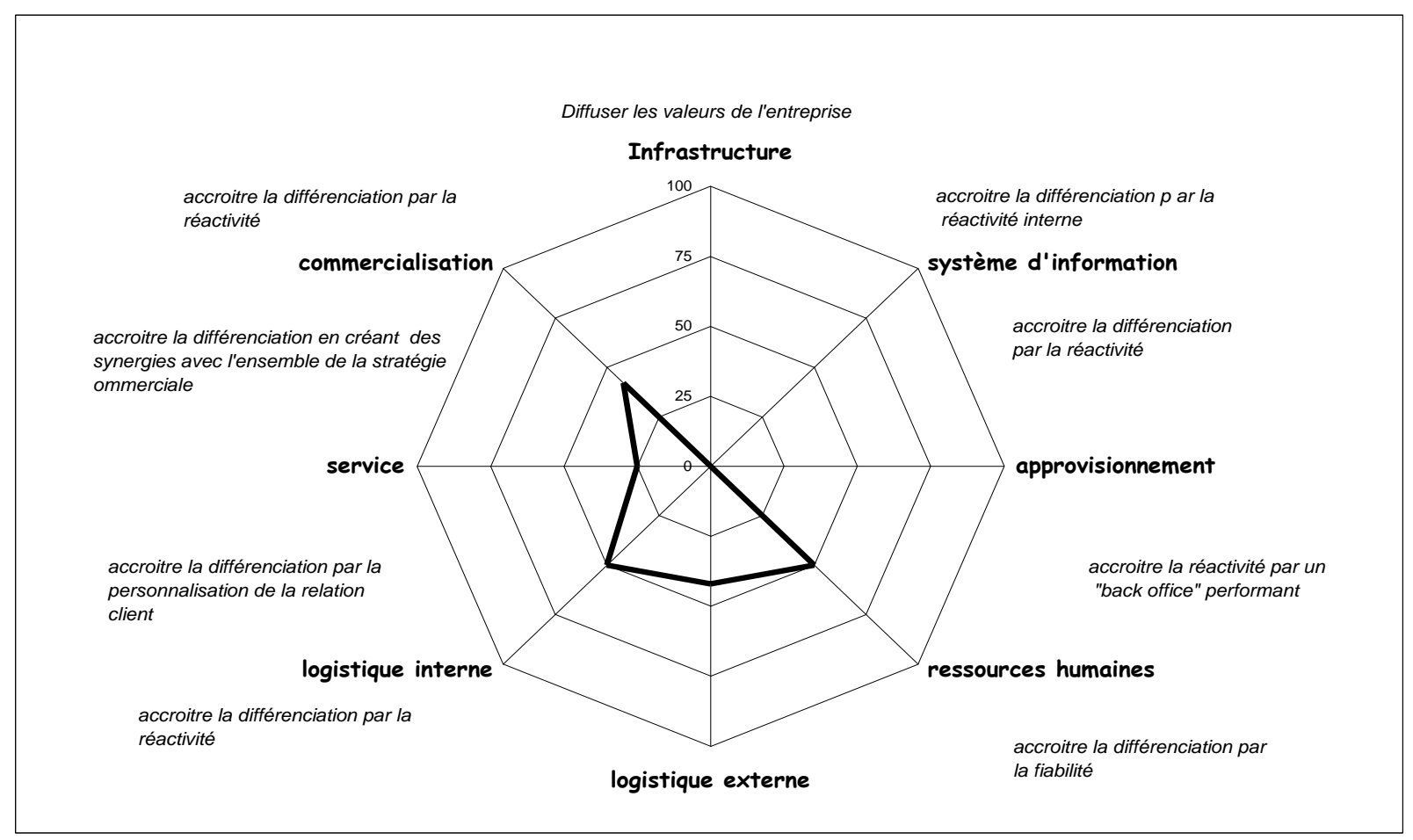

Le modèle permet de montrer, de façon concrète, au dirigeant de PH les incidences de l'absence

d'alignement stratégique. On observe un décalage de niveau de synergie entre certaines activités de la chaîne de valeur et le site marchand. Les auteurs du site ont négligé les liens avec trois des activités de soutien. Les recommandations peuvent être formulées dans ce sens.

\section{Conclusion}

Le recoupement des réflexions académiques ne masque pas l'absence d'un cadre pour aborder la problématique de l'intégration d'un site dans la stratégie et l'organisation. C'est pourquoi, à l'appui d'une démarche portérienne consistant à enrichir la chaîne de valeur d'une nouvelle activité, nous proposons un outil de mesure de l'intégration destiné à faciliter, pour le manager, la prise de conscience des forces et faiblesses de son organisation dans ce domaine.

Alors que les concepts évoqués dans la revue de littérature (alignement, impasse numérique etc) n’ont pas, à notre connaissance, été opérationnalisés, notre modèle présente l'intérêt de proposer un outil de synthèse 
pragmatique, synthétique et pédagogique. Il traduit les points de performance du site et attire l'attention sur les aspects organisationnels et stratégiques qui n'avaient pas été pris en compte jusque là. Testé et utilisé jusqu'à présent auprès de PME, il devra l'être auprès d'entreprises ou d'organisations plus importantes et complexes. L'introduction de variables significatives de la réflexivité des acteurs dans des situations complexes pourrait dynamiser son utilisation dans ces configurations entrepreneuriales. 


\section{Bibliographie}

F Bergeron, L.Raymond, A.M. Croteau, « Comportements stratégiques, choix et gestion des systèmes d'information : contribution à la performance », SIM n, Vol 6, p 5-26, 2001

F.D. Davis, , « Perceived usefulness, perceived ease of use and user acceptance of information technology » MIS quarterly, vol 13, n³, p389-400,1989

P.L. Dubois E. Vernette, «Editorial » Recherche et Applications en Marketing. 2, 2001

L.Flores P. Volle « Potentiel relationnel et impact du site de marque sur la performance marketing » Décisions Marketing, 40, Octobre Décembre p.39-50, 2005

A. Giddens, Les conséquences de la modernité, L'harmattan, Paris, 1994

P. Kotler B. Dubois D.Manceau, Marketing Management, Pearson Education,Paris 11ème Ed 2003

P Kotler, J.Dipak, M. Suvit, Le marketing en mouvement, Pearson Education, Paris, 2002

J.Lendrevie, J. Lévy, D.Lindon, Mercator Dalloz 7ème Ed, Paris, 2003

J. Lévy, «Impacts et enjeux de la révolution numérique sur la politique d'offre des entreprises » Revue Française de Marketing p.13-28, 2000.

E.W. McFarlan, J.L. Mckenney, P. Pyburn, « The information archipelago, plotting a course » Harvard Business Review, vol 61, n¹ p145-156,1983

Madrid C et MC.Monnoyer , La dimension numérique dans la stratégie commerciale, Ed L'harmattan, Paris, 2005

R.E. Miles et C.C. Snow, Organizational strategy,structure and process.Mc Graw Hill New York, 1978, J.F. Notebaert, « L'interface homme -machine en commerce électronique : vers une création de lien social comme outil de positionnement stratégique » Revue Française de Marketing p 71-90, décembre 5/5,2005

M. Porter et V. Millar, "Pour battre vos concurrents maitriser mieux l'information". Harvard l'Expansion printemps, p 6-20,1986

M.E Porter, «Strategy and the Internet » Harvard Business review, vol 79, n 3, mars 2001, p 63-78, 2001

S. Morton(ed), L'entreprise compétitive au futur, Les éditions d'organisation, 1995

S. Torger, « Origines et évolutions de l'interactivité dans la littérature marketing : de l'information à l'aide à la décision » Actes du Congrès de l'Association Française de Marketing, Nantes, 2006

R Vanheems, «Stratégie de distribution duale : proposition d'un instrument de mesure des transferts de clientèle inter-formules », Actes du Colloque Etienne Thill, La Rochelle, 2004

JF. Variot, «Le reengineering du marketing via le web”, L'Expansion Management Review, $\mathrm{n}^{\circ} 102$, septembre, p110-117, 2001

N. Venkatraman., J.C. Henderson , «Entreprise virtuelle cherche architecte », Expansion Management Review,mars p 6-17, 1999, 